\title{
A constitutive description of the anisotropic response of the fascia lata
}

\section{Citation}

Pancheri, F.Q., C.M. Eng, D.E. Lieberman, A.A. Biewener, and L. Dorfmann. 2014. “A Constitutive Description of the Anisotropic Response of the Fascia Lata." Journal of the Mechanical Behavior of Biomedical Materials 30 (February): 306-323. doi:10.1016/j.jmbbm.2013.12.002.

\section{Published Version}

doi:10.1016/j.jmbbm.2013.12.002

\section{Permanent link}

http://nrs.harvard.edu/urn-3:HUL.InstRepos:30782192

\section{Terms of Use}

This article was downloaded from Harvard University's DASH repository, and is made available under the terms and conditions applicable to Open Access Policy Articles, as set forth at http:// nrs.harvard.edu/urn-3:HUL.InstRepos:dash.current.terms-of-use\#OAP

\section{Share Your Story}

The Harvard community has made this article openly available.

Please share how this access benefits you. Submit a story.

Accessibility 


\title{
A constitutive description of the anisotropic response of the fascia lata
}

\author{
F. Q. Pancheri ${ }^{\mathrm{a}}$, C. M. Eng ${ }^{\mathrm{b}, \mathrm{c}}$, D. E. Lieberman ${ }^{\mathrm{c}}$, \\ A. A. Biewener ${ }^{\text {b }}$ L. Dorfmann ${ }^{\mathrm{d}, *}$ \\ ${ }^{a}$ Department of Mechanical Engineering \\ Tufts University, Medford, MA \\ ${ }^{b}$ Department of Human Evolutionary Biology \\ Harvard University, Cambridge, $M A$ \\ ${ }^{c}$ Department of Organismic and Evolutionary Biology \\ Harvard University, Cambridge, $M A$ \\ ${ }^{d}$ Department of Civil and Environmental Engineering \\ Tufts University, Medford, MA
}

\begin{abstract}
In this paper we propose a constitutive model to analyze in-plane extension of the fascia lata in goats. We first perform a histological analysis of the fascia that shows a well-organized bi-layered arrangement of undulated collagen fascicles oriented along two well defined directions. To develop a model consistent with the tissue structure we identify the absolute and relative thickness of each layer and the orientation of the preferred directions. New data are presented showing the mechanical response in uniaxial and planar biaxial extension. The main part of the paper focuses on a constitutive theory to describe the mechanical response. We provide a summary of the main ingredients of the nonlinear theory of elasticity and introduce a suitable strain-energy function to describe the anisotropic response of the fascia. In the final part of the paper we validate the model by showing good agreement of the numerical results and the experimental data.
\end{abstract}

Keywords: Connective tissue, fascia lata, histological analysis, uniaxial data,

\footnotetext{
${ }^{*}$ Corresponding author

Email address: Luis.Dorfmann@tufts.edu (L. Dorfmann )

$U R L:$ http://ase.tufts.edu/msml/ (L. Dorfmann )
} 
biaxial data, constitutive model

\section{Introduction}

Deep fascia is a type of connective tissue found throughout the body that encloses many muscles and connects them to each other and to bones. There is growing evidence that fascia can influence limb stability, force transmission, and elastic energy storage during locomotion (Garfin et al., 1981; Bennett, 1989; Maas and Huijing, 2005; Maas et al., 2005a,b). However, quantifying the role of fascia during active movement presents challenges. First, its sheetlike structure contains multiple layers of well-organized collagen fibers making the tissue highly anisotropic. Second, deep fascia has multiple connections to muscles and bones that generate complex non-homogeneous states of strain. The complex structure and loading environment of fascia may help explain why many current musculoskeletal models ignore its role in simulations of muscle function (see Tang et al. (2009) and Lee et al. (2010)). In an effort to address this deficiency, we describe here the mechanical properties of an example of a deep fascial structure, the fascia lata in the goat hind limb, using structurally-driven assumptions to capture and reproduce its anisotropic response measured during strain-controlled uniaxial and biaxial tension tests.

Most published data on the fascia lata have been limited to uniaxial tension with either the applied stretch increasing monotonically or in the form of periodic loading, unloading and reloading up to different, but fixed, stretches (Gratz, 1931; Butler et al., 1984; Bennett, 1989). Gratz (1931), using fresh human specimens of fascia lata, identifies the maximum safe stress in simple tension as $14.5 \mathrm{MPa}$ corresponding to a strain of $3.5 \%$ and the ultimate strength as $54 \mathrm{MPa}$ for $9 \%$ strain. A wide range of values of the tissue strength have been reported in literature (Hinton et al., 1992; Butler et al., 1984), likely due to methodological differences in measurement of the mechanical response (Butler et al., 1984; Sacks, 2000). Most biological tissues are anisotropic, requiring a wide range of experimental data to obtain appropriate representations of their behaviors. Strain-controlled planar biaxial tension can generate deformations that more closely resemble fascia's in vivo state of strain compared to uniaxial tension.

Fascia lata's structure has been described differently because of its layered arrangement and, possibly, because of variation between different species in which it has been studied. For example, Butler et al. (1984) described human fascia lata as a bi-layered tissue with weak interaction between layers. 
Based on analysis of fascia lata from a number of different mammals, Bennett (1989) distinguished three distinct layers: a thin outermost layer rich in randomly oriented collagen fibers and two inner layers each containing parallel fascicles of collagen fibers oriented along a clear direction. The two families of fibers each contained in one of the layers, enclose an angle of $70^{\circ}$ to 85. More recently, Stecco et al. (2009) proposed that deep fascia has either completely independent layers or some regions within layers that are loosely connected by bundles of collagen fibers present in low quantities. These varied descriptions of fascial structure highlight the importance of combining tissue-specific structural investigations and accurate experimental data towards constitutive modeling formulations.

Constitutive models (mathematical models of material behavior) of biological tissues have experienced a constant progression over the years in which the material structure has become the enabling criteria to perform simplifications of their three dimensional expressions. Initially, published phenomenological models of biological tissues focused on isotropic symmetry (Fung, 1967; Veronda and Westmann, 1970; Demiray, 1972; Haut and Little, 1972; Jenkins and Little, 1974). These formulations can properly replicate uniaxial data of biological tissues rich in collagen fibers, however, the isotropic symmetry assumption is questionable since it is inconsistent with the structure and mechanical response of the material. More recently, greater focus has been placed on evaluating structural characteristics of collagen-rich biological tissues in relation to the material anisotropic response and in merging the findings to develop new constitutive descriptions (Lanir, 1983; Sacks and Sun, 2003; Lin et al., 2009; Natali et al., 2010). A comprehensive review of different constitutive formulations that use exponential, polynomial and logarithmic forms of strain energy functions to describe anisotropy within the tissue are given by Holzapfel et al. (2000).

In the present study we develop a constitutive model of goat fascia lata, which assumes nonlinear elasticity and anisotropy. Our approach investigates the structure of fascia lata in goats containing oriented fascicles of wavy collagen fibers that are confined within two well-defined layers. In one layer, the fibers run longitudinally by inserting proximally into the tensor fascia lata and connecting distally to the patella and knee. The second, transverse layer contains fibers that span the tissue, connecting with the gluteobiceps muscle (see Figure 1). We develop the model to be consistent with the tissue structure after identifying the number, arrangement, absolute and relative thickness of individual layers. The tissue response is measured via strain- 
controlled biaxial testing, enabling us to capture its general behavior which results from the two primary families of fibers that are not mechanically equivalent or orthogonal. With this procedure we overcome previous limitations that focused on replicating only uniaxial experimental data of the tissue analytically and which did not properly account for the presence of more than one single family of fibers. The different loading conditions we apply here are required to obtain model parameters that are representative of the tissue behavior for a wider range of deformations. A more accurate constitutive model of fascia can be incorporated into musculoskeletal simulations and used to better understand the interaction between muscle and fascia during locomotion.

The paper is organized as follows. Section 2 describes the methods to characterize the tissue structure and the experimental procedure used to determine the stress-deformation response in vitro. Section 3 presents the images and parameters extracted from the structural characterization along with the stress-deformation response. Section 4 introduces the notation necessary to describe the kinematics with the appropriate stress and strain tensors along with their associated invariants. The hyperelastic constitutive model is developed in Section 5 and the corresponding numerical results are compared to data. Concluding remarks are provided in Section 6.

\section{Experimental Methods}

All experiments performed in this study met Harvard University and Tufts University IACUC guidelines. Fascia lata was dissected from the hindlimbs of five adult goats Capra hircus shortly after death and then wrapped in cotton gauze soaked with $1 \times$ phosphate buffered saline (PBS). Samples were maintained at $4^{\circ} \mathrm{C}$ prior to mechanical testing, which was completed within 48 hours post mortem.

\subsection{Tissue imaging and histological investigation}

Digital microscopic images of fascia lata samples were used to measure the angle enclosed by the longitudinal and transverse fascicles in their respective layers. The angle is defined from the contrast created by the edges of the parallel fiber bundles in each layer when imaged using an inverted light mi-

croscope (Figure 1b). After image acquisition, all analysis and measurements were performed with ImageJ (NIH). 


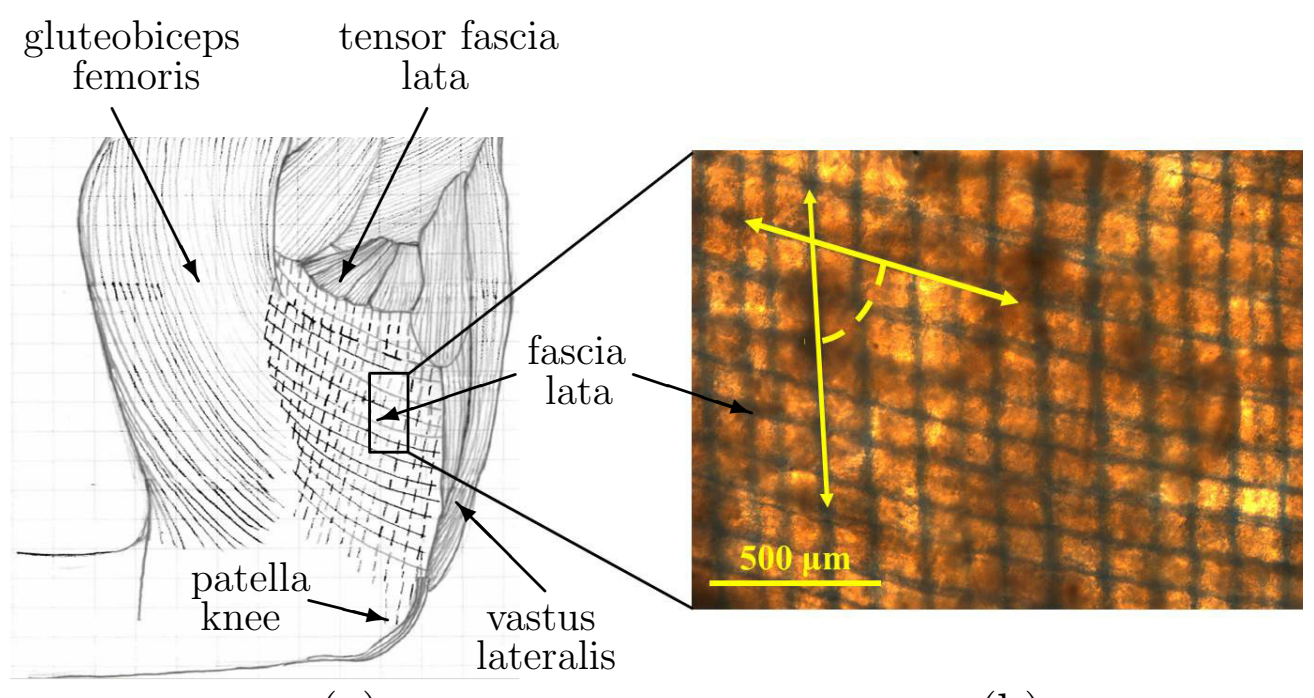

(a)

(b)

Figure 1: Image (a) shows the location of facia lata and major muscles groups in hindlimbs of adult goats (Bennett (1989), reproduced with permission of the copyright owner, Wiley, UK). Image (b), light microscopy, is used to determine the structural arrangement of the preferred directions. From the analysis of multiple samples we found that the angle between the longitudinally and transversely oriented collagen fibers is between $67^{\circ}$ and $80^{\circ}$.

Four small strips of tissue approximately $10 \mathrm{~mm} \times 15 \mathrm{~mm}$ were fixed in $3.65 \%$ formaldehyde solution before being embedded in paraffin. For the histological analysis we prepared $5 \mu \mathrm{m}$ thick sections from each strip of material taken at four locations along their length. Two sets of sections were prepared. The first set was obtained by cutting the tissue along a plane perpendicular to the longitudinal fiber direction, the second by cutting a different sample perpendicular to the transverse fiber direction. For consistency, a light microscope was used to identify the fiber directions prior to paraffin embedding. Sectioned samples were mounted on slides and stained with Hematoxylin and Eosin (H\&E) to obtain an overall inclusive image of the tissue sample, and Masson's trichrome to stain collagen fibers and tissue matrix. Slides were examined at $50 \times$ using a Zeiss Axiovert 40 CFL inverted microscope with standard dichromic mirrors.

The digital image collection of the slides was performed with integrated QCapture Pro software (QImaging, Surrey, BC, Canada). First, a global calibration scale was applied by imaging a stage micrometer. Next, images captured using the same length scale, were analyzed to determine the thickness of each layer and the cross sectional area ratio of longitudinal to transverse fascicles. A threshold was set prior to conversion into binary mode 
such that only the collagen fascicles were visible. Images were converted to an 8-bit grayscale format to differentiate the mature fiber fascicle from the loose collagen ground matrix surrounding them. The cross sectional area of each bundle was then extracted and individually identified as longitudinal or transverse prior to summation to calculate the total cross sectional area of the bundles in each layer. The thickness of the layers was measured manually by taking 64 individual equi-spaced measurements per layer per slide.

\subsection{Sample preparation for mechanical testing}

Using a custom made cruciform die cutter, two samples were obtained from the fascia lata of each goat leg. The two arm pairs of the die cutting tool have a width of $12 \mathrm{~mm}$ each and measure $62 \mathrm{~mm}$ from end to end, see Figure 2a. The gage region is precluded by the $12 \mathrm{~mm} \times 12 \mathrm{~mm}$ square area in the center of the cruciform where four physical gage marks were attached with Loctite 4013 medical device adhesive. The marks were used by the machine for measuring deformation and controlling strain rate. Prior to testing, the terminal parts of the sample ends were wrapped with 800 grit sand paper and securely clamped in custom designed grips. Fine sand paper reduces the risk of slip and optimizes distribution of the clamping pressure across the specimen arm to decrease the risk of localized damage.

Although the same die was used to cut all cruciform samples, some variations in the final shape occur due to the slippery texture of the tissue in the hydrated state. Therefore, an image of the sample from the video extensometer was recorded before testing and used later to measure the gage region dimension of each sample (Figure 2a) to calculate cross sectional area. Similarly, the constant strain rate in all samples was ensured by imposing a deformation speed that was proportional to the distance between opposing marks measured by the video extensometer in the reference configuration. The average thickness of the gage region of each sample was measured using a contact micrometer (Mitutoyo Corp.) with a resolution of $\pm 0.01 \mathrm{~mm}$. In addition to the cruciform specimens, longitudinally and transversely oriented rectangular strips of fascia lata were cut for uniaxial tension tests.

Two mutually orthogonal in-plane directions with the convention that the fiber and loading direction always coincide along the 2-axis of the cruciform sample (Figure 2b) were defined to outline the test sequence and data evaluation. Therefore, for each fascia lata, one sample was cut with the longitudinal set of fibers closely aligned with one of the axes of the cutting die (2-axis), in the second sample the 2-axis was aligned with transverse fibers. 
The sketch in Figure $2 \mathrm{~b}$ shows that one of the fiber direction does not coincide with the directions of the applied load. This condition generates a nonhomogeneous deformation within the gage region of the specimen and has important implications in material modeling (Holzapfel and Ogden, 2009).

\subsection{Mechanical testing}

All mechanical testing was performed using a custom built Zwick/Roell planar biaxial testing machine. The machine controls strain measured from the specimen in real time to within a tolerance of $0.25 \%$ by independently modulating the applied load at each actuator drive. Details and layout of the equipment are given in Pancheri and Dorfmann (2013).

To assess the mechanical properties of fascia lata in biaxial tension we programmed the equipment to perform two series of tests where a new sample was used at the start of each series. The applied maximum stretch levels were designed to generate a strain field in the tissue sample that nears but is below its damage point. The collagen fibers of fascia lata, which are oriented longitudinally, coincide with the loading-unloading direction (2-axis) in the first series. During the second series, the fibers that span transversely across the tissue were aligned with the loading direction. The reference configuration was determined at the beginning of each testing sequence by the position of the physical gage marks when a $0.08 \mathrm{~N}$ preload was detected by all four load cells. This value was the minimum to assure the samples maintain the horizontal position prior to immersion in the water bath and was critical to ensure the same initial conditions for all tests. Testing was conducted with samples immersed in a constant temperature bath at $39^{\circ} \mathrm{C}$, controlled by an auto regulated circulator (Ecoline Type RE 104; LAUDA Königshofen, Germany). A quasi-static strain rate of $0.0015 \mathrm{~s}^{-1}$ was applied throughout all tests. Each test series contained a sequence of four biaxial loading routines, with each routine followed by a $180 \mathrm{~s}$ rest period. Upon completion of each sequence the sample was always returned to the initial reference configuration. The test series was as follows:

- Starting from the reference configuration, with $\lambda_{1}=1$ held constant, the specimen was subjected to five loading-unloading cycles in the 2direction with maximum extension $\lambda_{2}=1.04$.

- After the rest period, with $\lambda_{2}=1$, the specimen was first stretched to $\lambda_{1}=1.03$. Then, with $\lambda_{1}=1.03$ held constant, the specimen 
was subjected to five loading-unloading cycles with maximum stretch $\lambda_{2}=1.04$.

- Again, after the rest period, with $\lambda_{1}=1$ held constant, the specimen was then subjected to five loading-unloading cycles with maximum extension $\lambda_{2}=1.06$.

- Once more, after the rest period, $\lambda_{1}$ was increased to 1.03 and kept constant, the specimen was subjected to five loading-unloading cycles with maximum stretch $\lambda_{2}=1.06$.

To determine the values of the parameters of the constitutive model we averaged the biaxial extension data corresponding to the loading phase of the last cycle of each sequence. Averaging the response of the samples from five different animals, reduces inter-sample variations and makes the model parameters representative of the tissue rather than individual samples.

The rectangular specimens used to determine the mechanical response in uniaxial tension underwent a single extension to rupture. Six samples collected from the five goats were stretched in the direction of the longitudinally oriented collagen fibers. Six additional samples from 3 of the goats were subjected to simple tension in the direction of the transversely oriented fibers. Similarly to the cruciform samples, using stretch as the independent variable, the uniaxial tension data were averaged to characterize the tissue response in the longitudinal and transverse directions.

\section{Experimental Results}

The value of the opening angle between the longitudinal and transverse fiber directions is determined to be between $67^{\circ}$ and $80^{\circ}$, see Figure 1.

\subsection{Histology}

Histological sections show that the fascia lata has a well-organized structure comprising oriented fascicles with interspersed fibroblasts and fibrocytes, see Figure 3a. Fibrocytes play a vital role to maintaining the overall structural integrity of the tissue in the living animal. Undulated fibers of mature Type I collagen form the bundles, see Figure 3b (Masson's Trichrome).

The structure of fascia lata is bi-layered, with the proteoglycan (ground) matrix connecting the layers and enveloping all fascicles. Although collagen is present in the matrix, it is less organized and thus likely allows for some 


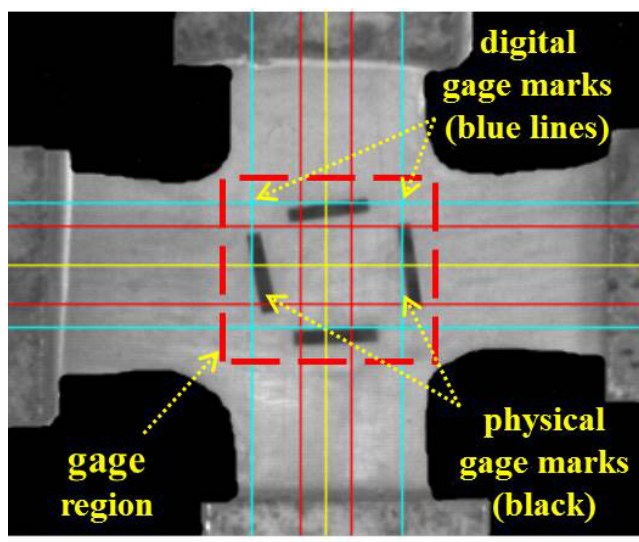

(a)

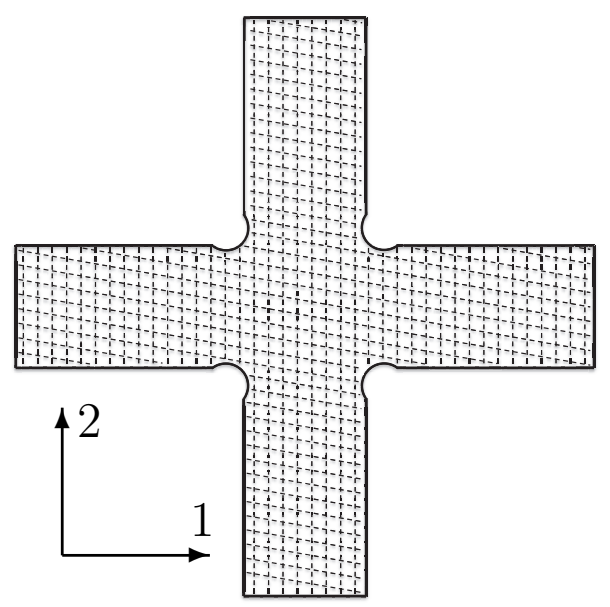

(b)

Figure 2: (a) A cruciform shaped specimen in biaxial tension. Using the provided contrast, the digital gage marks follow the movement of the physical markers. Each gage mark is tracked independently, which allows to control and adjust the strain rate of each actuator. The Cartesian coordinate system used to describe the test sequence and the orientation of the preferred directions are shown by the schematic representation (b).
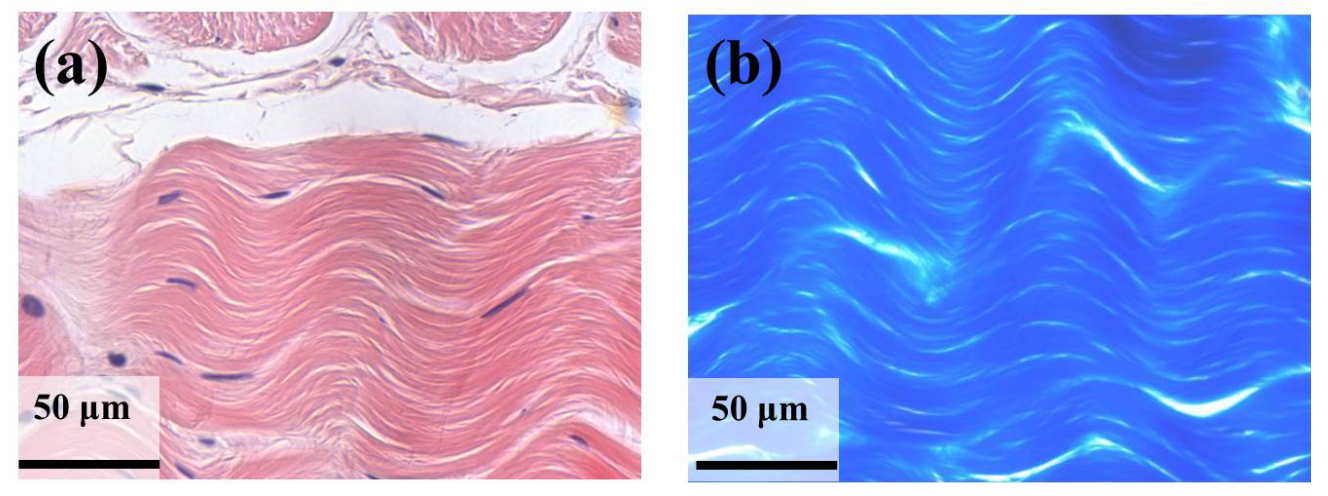

Figure 3: (a) The well-organized structure of fascia lata contains many interspersed fibroblasts (H\&E stain $400 \times$ magnification). (b) The fascicles of Type I collagen contain undulated collagen fibers (Masson's Trichrome stain 400× magnification).

degree of motion between individual fascicles and between the two layers of tissue. Figure 4a shows a section obtained by cutting fascia lata perpendicular to the transverse fiber direction, Figure $4 \mathrm{~b}$ shows a section obtained by cutting the tissue perpendicular to the longitudinal fibers. The arrangement of longitudinally and transversely oriented fascicles is distinctly different. The uppermost layer contains the transversely oriented fibers which are grouped in a single row of fascicles; the lower layer contains the longitudi- 

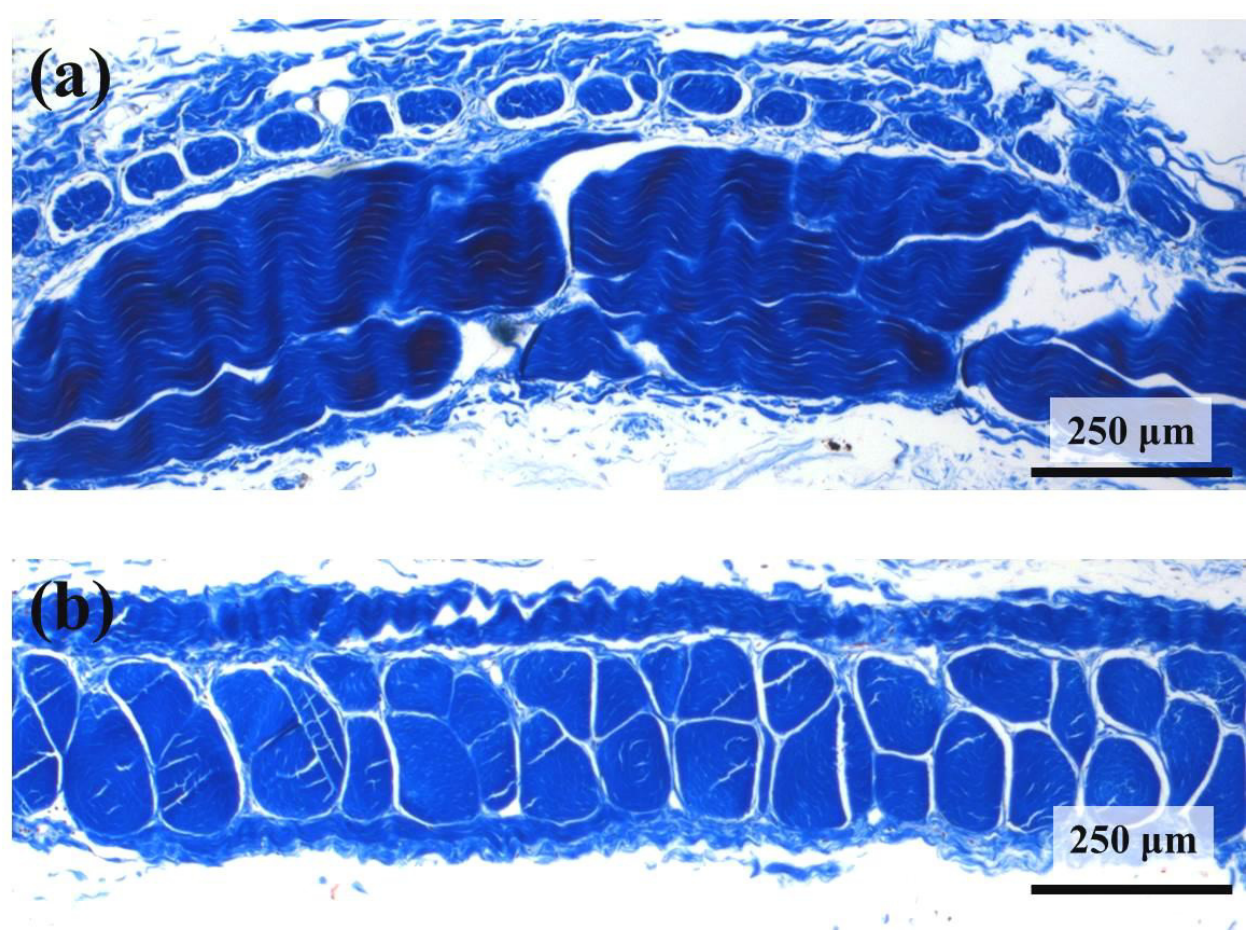

Figure 4: Histological sections of the tissue at 50× magnification. Image (a) shows a section that is obtained by cutting fascia lata perpendicular to the transverse fiber direction, image (b) by cutting the tissue perpendicular to the longitudinal fibers. The layer of longitudinal fascicles is on average 4 times thicker than the transverse.

nally oriented fibers. Histology slides show that the cross sectional area of fascicles in the longitudinal direction are generally always larger in size than those of the transverse. In some regions, one large fascicle spans the entire thickness of the longitudinal layer; in others, two or even three of lesser diameter are present across its thickness. The thickness of the longitudinally and transversely oriented fiber layers is $246.4 \pm 42.8 \mu \mathrm{m}$ (mean \pm S.D.) and $61.3 \pm 8.7 \mu \mathrm{m}$, respectively. We find that, using images captured from slides at the same length scale, the total cross sectional area ratio of longitudinal to transverse fascicles is between 3.6 and 5.7.

\subsection{Mechanical characterization}

\subsubsection{Simple tension}

Specimens subject to simple tension reach maximum stress when $\lambda$ is between 1.1 and 1.18. The Cauchy stress at failure is $38.4 \pm 10.3 \mathrm{MPa}$ (mean 


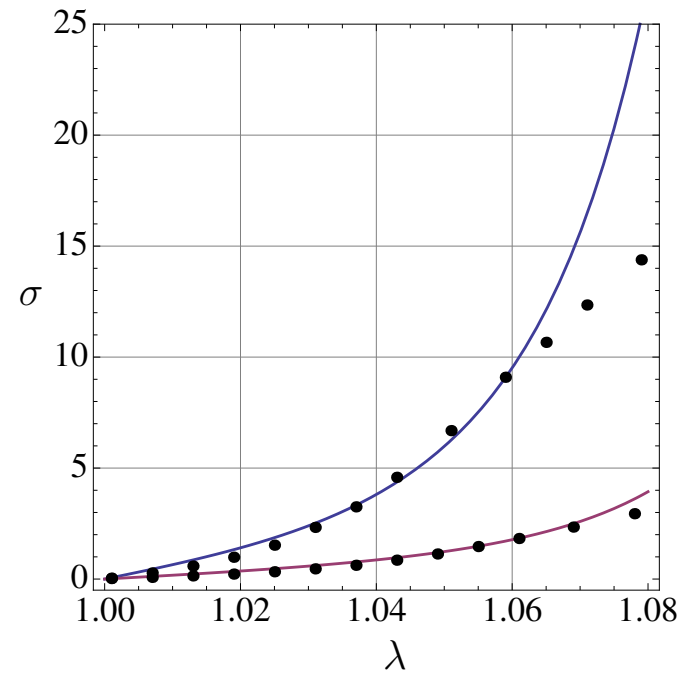

Figure 5: Cauchy stress $\sigma$ versus stretch $\lambda$ of fascia lata in uniaxial tension in the direction of longitudinally and transversely oriented fibers. The dotted lines represent averaged experimental data, the solid lines the corresponding numerical results. Stress values are given in MPa.

\pm S.D.) in the longitudinal direction and $8.6 \pm 2.9 \mathrm{MPa}$ in the transverse direction. Since the model parameters are obtained from the biaxial data following the procedure outlined in Section 2.3, for ease of comparison we restrict representation of the uniaxial stress-deformation response to $\lambda=1.08$ (see Figure 5). The uniaxial tension data are averaged and are shown by the dotted lines in Figure 5 as Cauchy stress versus stretch. The data clearly show that the mechanical response of specimens stretched in the direction of the longitudinally oriented fibers is distinctly stiffer compared to the response of samples that span transversely across fascia lata. The solid lines in Figure 5 represent the numerical results of the constitutive model and will be discussed in Section 5.1. The data show an exponential increase in stress up to an elongation of approximately $\lambda=1.06$ at which point permanent damage occurs in the material.

\subsubsection{Biaxial tension}

In this section we present representative results of the stress-deformation response of a longitudinal and a transversely oriented sample based on the protocol in Section 2.3. A detailed analysis of material properties will be provided in a forthcoming publication (Eng et al. (2013)). 
Figures 6 and 7 show the response of a specimen in which the longitudinally oriented fibers of fascia lata coincide with the 2-axis. Specifically, the figures display the Cauchy stress in the 2-direction as a function of stretch for two different, but constant extensions in the 1-direction, see Figure 2b for the orientation of the coordinate axes. Similarly, Figures 8 and 9, present load-deformation results in biaxial tension using an experimental setup where transversely oriented fibers of fascia lata coincide with the 2-axis. In all cases five loading-unloading cycles in the 2-direction are performed. For clarity only the last cycle is shown.

Images (a) and (c), in Figures $6-9$ depict the response during initial extension in the 1 -direction to a pre-selected value $\lambda_{1}$ with $\lambda_{2}=1$ constant. Now, with $\lambda_{1}$ held constant, the specimen is subjected to loading-unloading cycles in the 2-direction with maximum values of $\lambda_{2}=1.04$ and $\lambda_{2}=1.06$.

The images denoted (a) and (b) in Figure 6 depict the stress-stretch responses of the loading-unloading sequence in the 2 -direction with maximum stretch $\lambda_{2}=1.04$ and with no deformation in the 1-direction $\left(\lambda_{1}=1\right)$. The images $(\mathrm{c})$ and $(\mathrm{d})$ show the loading-unloading in the 2-direction with the same maximum stretch $\lambda_{2}=1.04$, but $\lambda_{1}$ now increased to 1.03 . The same specimen, after a resting time of 180 seconds, was subject to additional characterization tests. The additional sequence was identical to the one shown in Figure 6, except that the maximum extension in the 2-direction was increased from $\lambda_{2}=1.04$ to $\lambda_{2}=1.06$. The corresponding results are shown in Figure 7.

The biaxial sequence was then used to determine the stress-stretch response of a different specimen, in which fibers that span the fascia lata were aligned with the loading-unloading direction (see Figure 2b). The results corresponding to the loading-unloading cycle in the 2-direction, with maximum stretches of 1.04 and 1.06, are shown in Figures 8 and 9. The extension in the 1-direction was again held constant at values of $\lambda_{1}=1$ and $\lambda_{1}=1.03$.

Fascia lata is much stiffer in the longitudinal direction compared to the transverse direction. This difference can be seen, in Figures 6 and 8 by comparing values of the Cauchy stress $\sigma_{2}$ during cyclic loading as represented in the images (b) and (d) (Note the change in scale from Figures 6 to 8). The same observation applies to the Cauchy stress $\sigma_{2}$ in Figures 7 and 9 shown in (b) and (d).

The Cauchy stress $\sigma_{2}$ during cycling loading in the images (b) and (d) in Figures 6 and 7 also shows that stretching fascia lata in the transverse direction does have a negligible effect on its response in the longitudinal direction. 
This differs from the results given in Figures 8 and 9, in which Cauchy stress $\sigma_{2}$ during cyclic loading in the transverse direction was affected more by the different amounts of stretch applied in the 1-direction. Further, the graphs in image (d) show that the stress required to maintain the stretch of 1.03 constant in the 1-direction is much higher than the stress required to cycle the tissue in the 2-direction. The biaxial response of the two samples highlight the observed mechanical anisotropy of fascia lata to an extent never captured through uniaxial tests alone. The tissue structure results are consistent with the biaxial response, in which the thicker collagen fiber layer in the longitudinal direction reinforces the tissue to withstand higher loads.

\section{Constitutive modeling}

The images of the tissue structure in Section 3.1 show that goat fascia lata consists of two distinct layers of different thickness containing fibers that are oriented along two non-orthogonal directions and are surrounded by a matrix containing collagen fibers of random arrangement. Therefore, not surprisingly, the measured planar biaxial response is highly anisotropic. Moreover, the response in the direction of the two fibers is not mechanically equivalent. Shear stresses during deformation are then unavoidable to maintain pure homogeneous deformation, regardless of the angle of the fibers relative to the Cartesian axes of strain. Also, the initial loading and subsequent unloading stress-stretch response curves, do not coincide indicating dissipation of energy. In the theory that follows, the material is considered anisotropic and nonlinearly elastic. The objective is to propose a constitutive model that accounts for the observed material behavior. We focus on the quasi-static response and consider the material properties and deformation to be independent of time. We define the notation necessary to describe the kinematics, summarize appropriate strain and stress tensors along with the associated invariants. For a more general representation of the theory we refer to, for example, Holzapfel (2001) and Ogden (1997).

\subsection{Basic equations}

To describe the deformation of a deformable body we select an arbitrary chosen configuration as a reference, which we denote $\mathcal{B}_{\mathrm{r}}$, and identify a generic material point by its position vector $\mathbf{X}$ relative to some origin. Application of mechanical forces deforms the body, so that the point $\mathbf{X}$ occupies the new position $\mathbf{x}=\boldsymbol{\chi}(\mathbf{X})$ in the deformed configuration $\mathcal{B}$. The vector field $\chi$ 

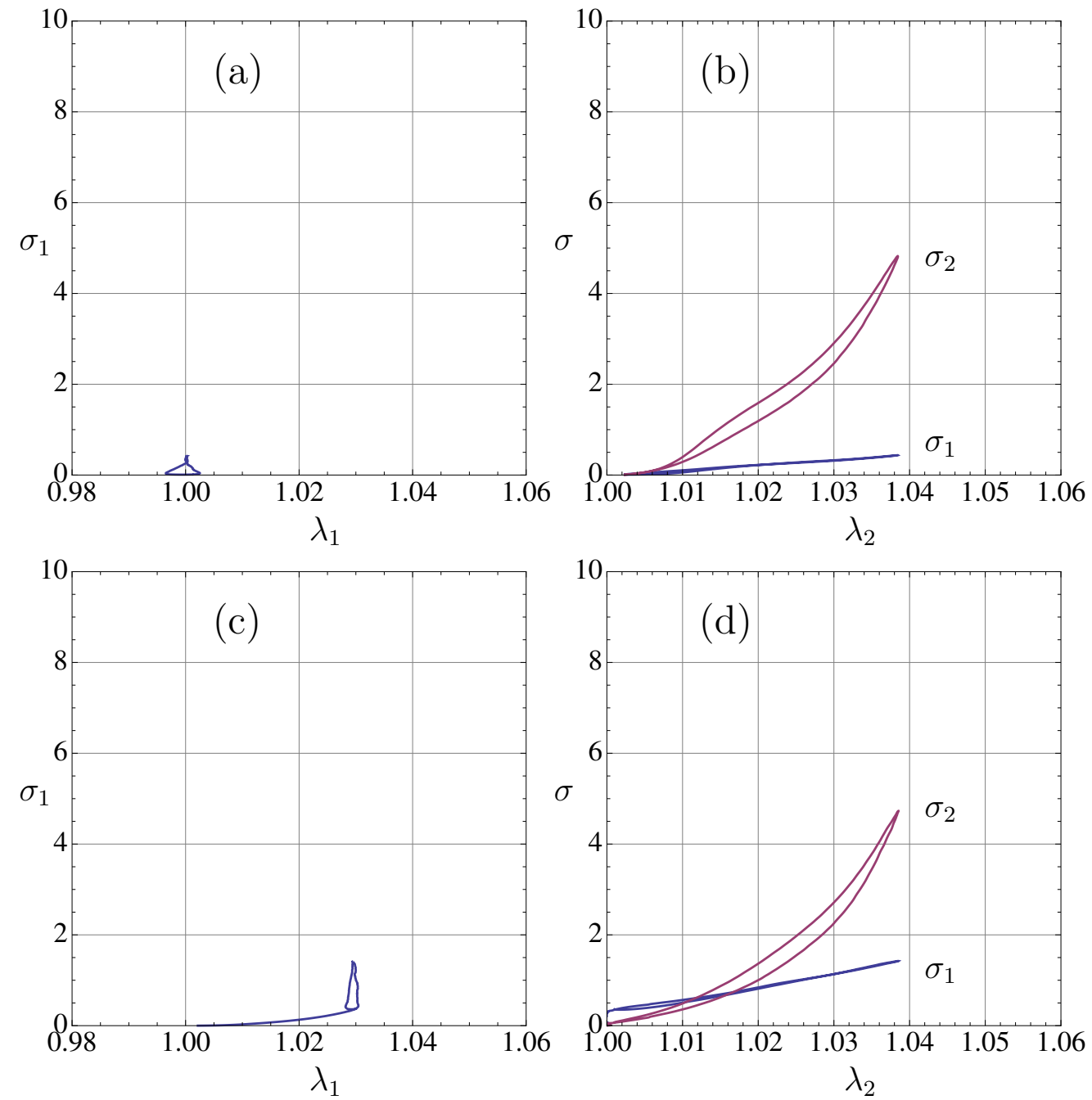

Figure 6: (a) and (c) depict the initial response of a preconditioned sample during extension in the 1-direction to a pre-selected value $\lambda_{1}$ with $\lambda_{2}=1$ constant. Then, with $\lambda_{1}$ held constant, the specimen is subjected to loading-unloading cycles in the direction of the longitudinally oriented fibers (2-direction) with maximum stretch $\lambda_{2}=1.04$. Graphs in (b) and (d) show the last of five cycles. Stress values are given in MPa.

describes the deformation of the body and assigns to each point $\mathbf{X}$ a unique position $\mathbf{x}$ in $\mathcal{B}$ and viceversa attributes a unique reference position $\mathbf{X}$ in $\mathcal{B}_{\mathrm{r}}$ to each point $\mathbf{x}$. The deformation gradient tensor $\mathbf{F}$ relative to $\mathcal{B}_{\mathbf{r}}$, is defined by

$$
\mathbf{F}=\operatorname{Grad} \mathbf{x}
$$



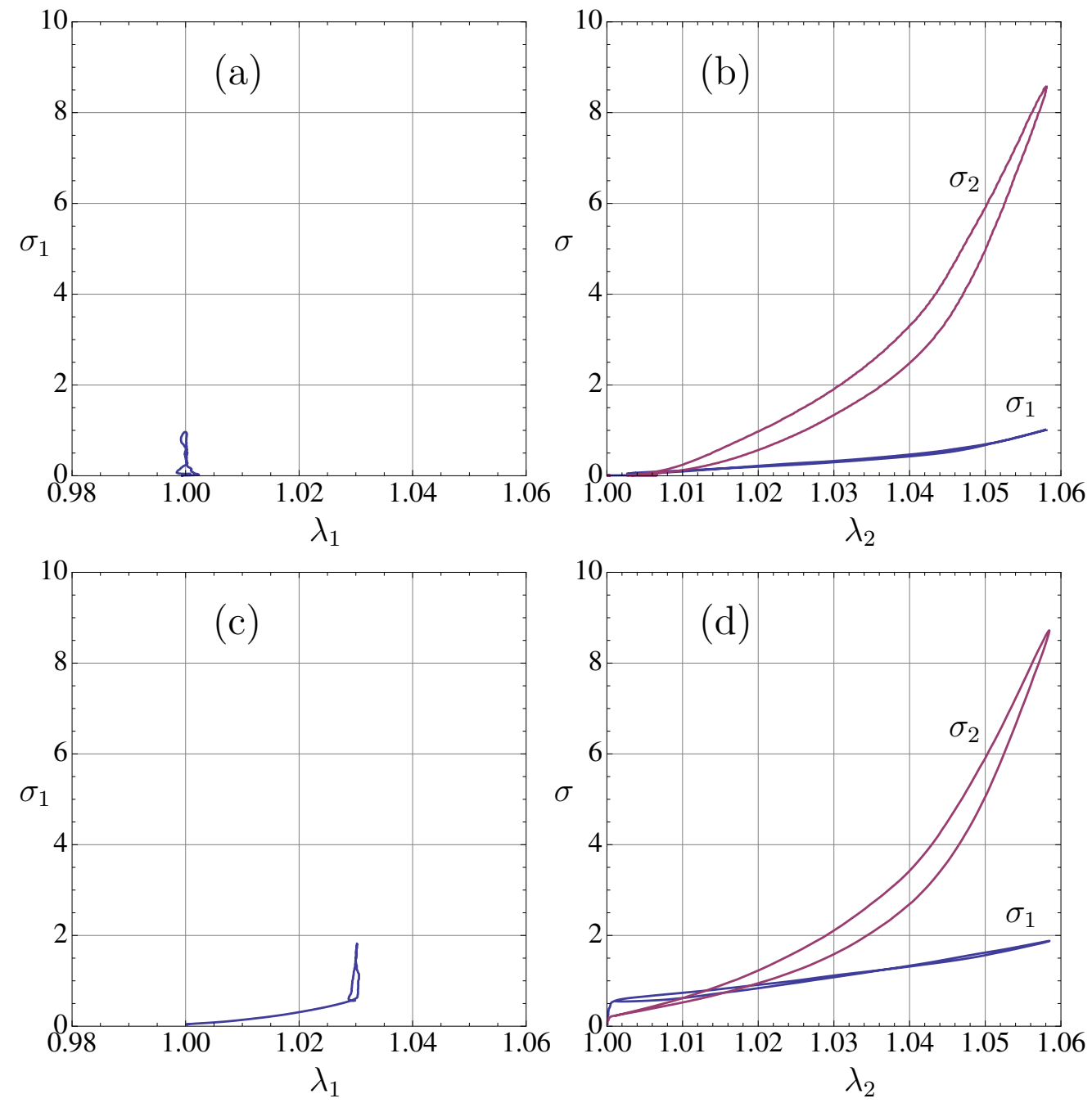

Figure 7: (a) and (c) depict the initial response of a preconditioned sample during extension in the 1-direction to a pre-selected value $\lambda_{1}$ with $\lambda_{2}=1$ constant. Then, with $\lambda_{1}$ held constant, the specimen is subjected to cyclic loading-unloading in the direction of the longitudinally oriented fibers (2-direction) with maximum stretch $\lambda_{2}=1.06$. Graphs in (b) and (d) show the last of five cycles. Stress values are given in MPa.

Grad being the gradient operator with respect to $\mathbf{X}$. The Cartesian components are $F_{i j}=\partial x_{i} / \partial X_{j}$, where $x_{i}$ and $X_{j}$ are the components of $\mathbf{x}$ and $\mathbf{X}$, respectively, with $i, j \in\{1,2,3\}$. We also adopt the standard notation $J=\operatorname{det} \mathbf{F}$, with the convention $J>0$.

The deformation referred to as planar extension is defined by the equa- 

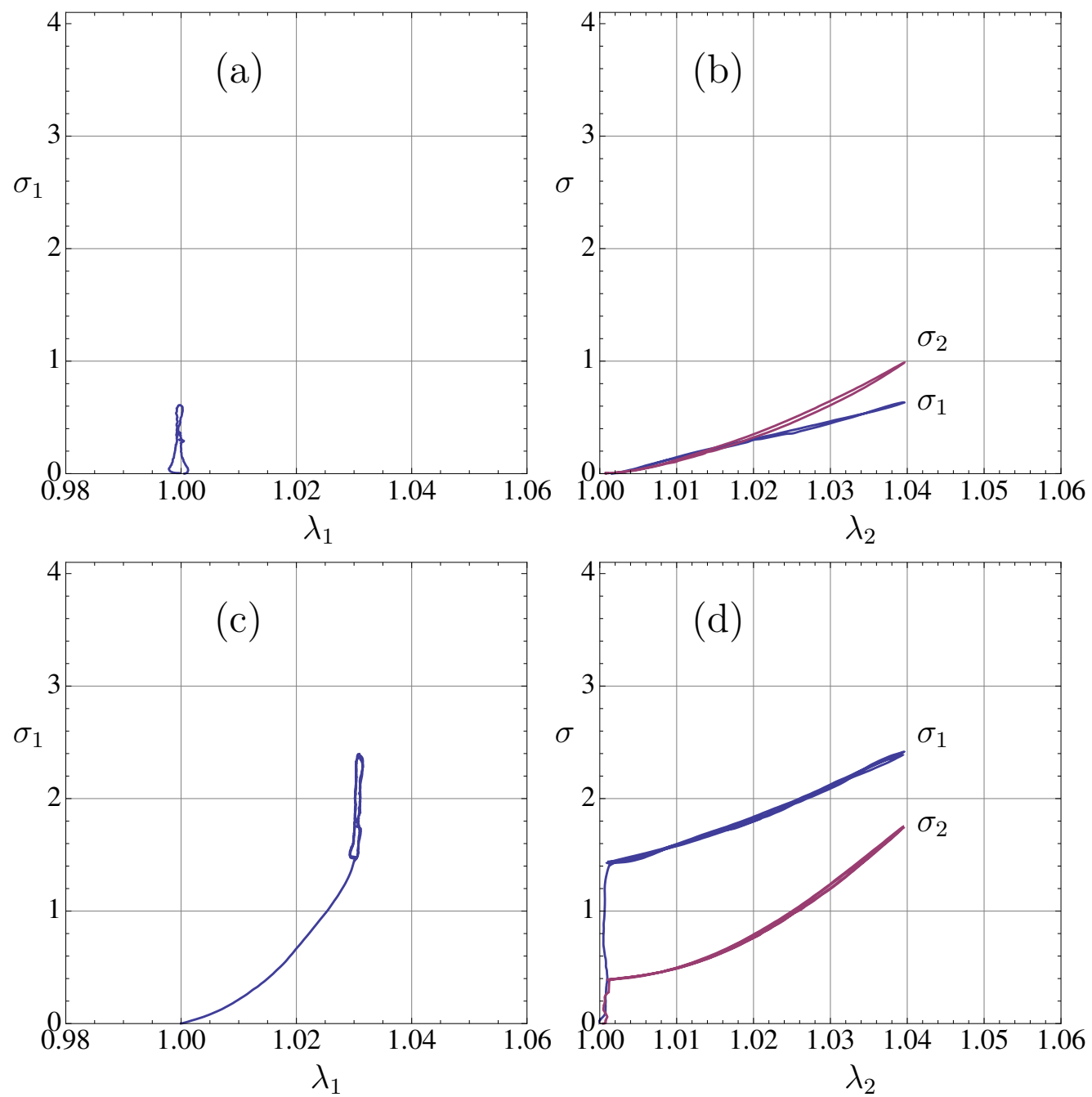

Figure 8: Graphs in (a) and (c) depict the initial response of a preconditioned sample during extension in the 1-direction to a pre-selected value $\lambda_{1}$ with $\lambda_{2}=1$ constant. Then, with $\lambda_{1}$ held constant, the specimen is subjected to cyclic loading-unloading in the direction of the transversely oriented fibers (2-direction) with maximum stretch $\lambda_{2}=1.04$. Graphs in (b) and (d) show the last of five cycles. Stress values are given in MPa.

tions

$$
x_{1}=F_{11} X_{1}+F_{12} X_{2}, \quad x_{2}=F_{22} X_{2}+F_{21} X_{1}, \quad x_{3}=F_{33} X_{3},
$$

where $F_{33}$ is the stretch in the out of plane direction, which we denote by $\lambda_{3}$. From these expressions we find that the components of the deformation gra- 

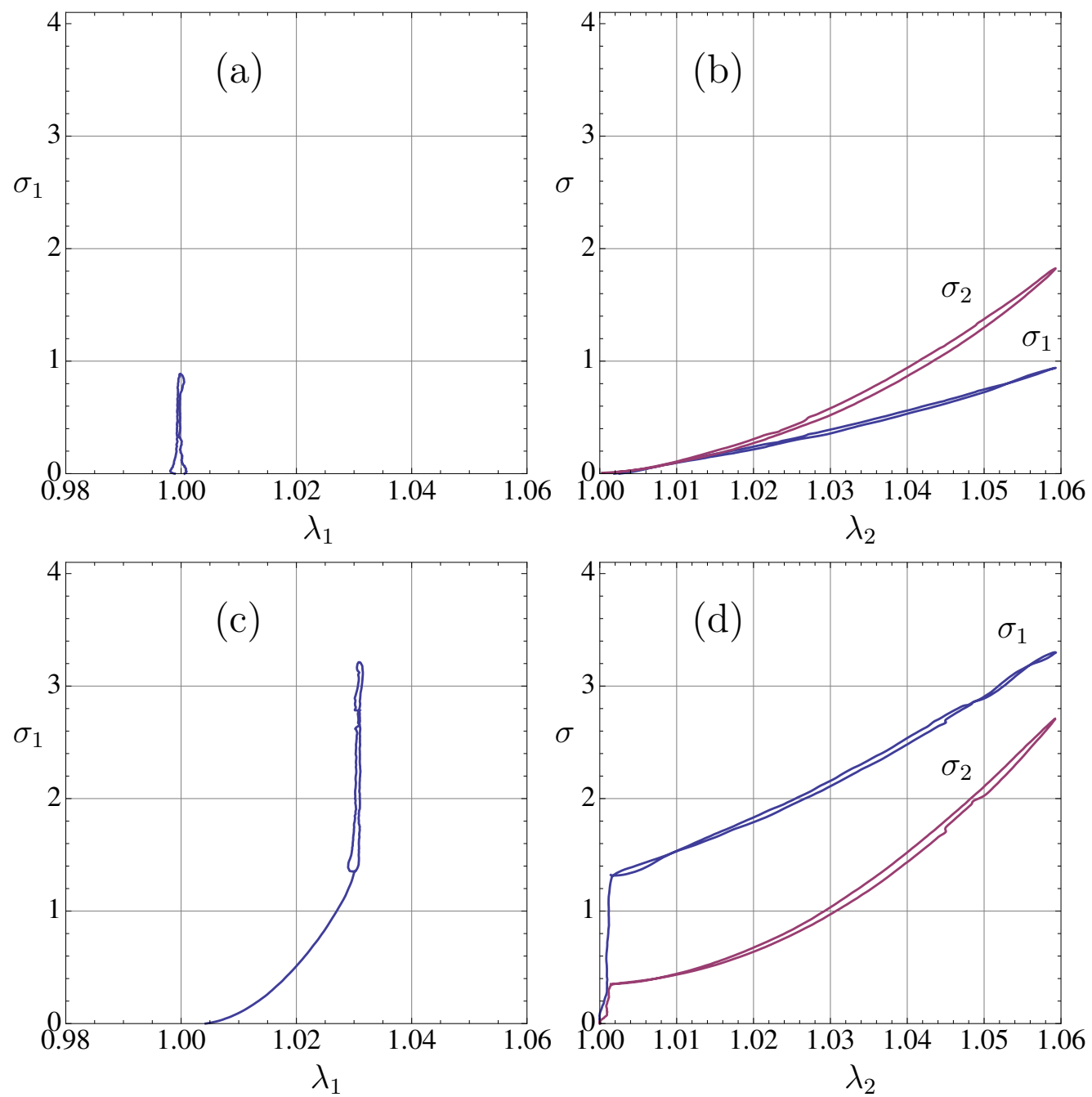

Figure 9: Graphs in (a) and (c) depict the initial response of a preconditioned sample during extension in the 1-direction to a pre-selected value $\lambda_{1}$ with $\lambda_{2}=1$ constant. Then, with $\lambda_{1}$ held constant, the specimen is subjected to cyclic loading-unloading in the direction of the transversely oriented fibers (2-direction) with maximum stretch $\lambda_{2}=1.06$. Graphs in (b) and (d) show the last of five cycles. Stress values are given in MPa.

dient $\mathbf{F}$, referred to the two sets of Cartesian coordinate axes, are represented by the matrix $F$, which is given by

$$
\mathbf{F}=\left[\begin{array}{ccc}
F_{11} & F_{12} & 0 \\
F_{21} & F_{22} & 0 \\
0 & 0 & F_{33}
\end{array}\right] .
$$


The resulting matrices of the left and right Cauchy-Green deformation tensors $\mathbf{B}=\mathbf{F F}^{\mathrm{T}}$ and $\mathbf{C}=\mathbf{F}^{\mathrm{T}} \mathbf{F}$, denoted $\mathrm{B}$ and $\mathrm{C}$, are

$$
\mathrm{B}=\left[\begin{array}{ccc}
F_{11}^{2}+F_{12}^{2} & F_{12} F_{22}+F_{21} F_{11} & 0 \\
F_{12} F_{22}+F_{21} F_{11} & F_{22}^{2}+F_{21}^{2} & 0 \\
0 & 0 & F_{33}^{2}
\end{array}\right],
$$

and

$$
\mathrm{C}=\left[\begin{array}{ccc}
F_{11}^{2}+F_{21}^{2} & F_{12} F_{11}+F_{21} F_{22} & 0 \\
F_{12} F_{11}+F_{21} F_{22} & F_{22}^{2}+F_{12}^{2} & 0 \\
0 & 0 & F_{33}^{2}
\end{array}\right]
$$

The theory of hyperelasticity characterizes the elastic response of a body by a strain energy function $W$ defined per unit volume in the reference configuration $\mathcal{B}_{\mathrm{r}}$. For a homogeneous material $W$ depends only on the deformation gradient $\mathbf{F}$ and we write $W=W(\mathbf{F})$. In this paper we restrict attention to incompressible materials, subject to the constraint $J=\operatorname{det} \mathbf{F} \equiv 1$. The nominal stress tensor $\mathbf{S}$ and the symmetric Cauchy stress tensor $\boldsymbol{\sigma}$ are then given, respectively, by

$$
\mathbf{S}=\frac{\partial W}{\partial \mathbf{F}}-p \mathbf{F}^{-1}, \quad \boldsymbol{\sigma}=\mathbf{F} \frac{\partial W}{\partial \mathbf{F}}-p \mathbf{I},
$$

where $p$ is an arbitrary hydrostatic pressure. Equation (5) shows that for an incompressible material, the Cauchy stress $\boldsymbol{\sigma}$ and the nominal stress $\mathbf{S}$ are related by $\boldsymbol{\sigma}=\mathbf{F S}$.

We introduce unit vectors $\mathbf{M}$ and $\mathbf{M}^{\prime}$ to denote the two preferred directions of an anisotropic material in the reference configuration $\mathcal{B}_{\mathrm{r}}$ and, following the work by Spencer (1971) and Ogden (2001), define the structural tensors $\mathbf{M} \otimes \mathbf{M}$ and $\mathbf{M}^{\prime} \otimes \mathbf{M}^{\prime}$. The material considered here is said to be isotropic if $W$ is an isotropic function of the three tensors $\mathbf{F}, \mathbf{M} \otimes \mathbf{M}$ and $\mathbf{M}^{\prime} \otimes \mathbf{M}^{\prime}$. Then, the form of $W$ is reduced to the dependence on the principal invariants $I_{1}, I_{2}, I_{3}$ of $\mathbf{C}$, which are defined by

$$
I_{1}=\operatorname{tr} \mathbf{C}, \quad I_{2}=\frac{1}{2}\left[I_{1}^{2}-\operatorname{tr}\left(\mathbf{C}^{2}\right)\right], \quad I_{3}=\operatorname{det} \mathbf{C}=J^{2},
$$

together with five invariants that depend on $\mathbf{M}$ and $\mathbf{M}^{\prime}$. These are calculated as

$I_{4}=\mathbf{M} \cdot(\mathbf{C M}), \quad I_{5}=\mathbf{M} \cdot\left(\mathbf{C}^{2} \mathbf{M}\right), \quad I_{6}=\mathbf{M}^{\prime} \cdot\left(\mathbf{C M}^{\prime}\right), \quad I_{7}=\mathbf{M}^{\prime} \cdot\left(\mathbf{C}^{2} \mathbf{M}^{\prime}\right)$, 
with the remaining invariant $I_{8}$ a function of both fiber directions

$$
I_{8}=\mathbf{M} \cdot\left(\mathbf{C M}^{\prime}\right) .
$$

For an incompressible anisotropic material the symmetric Cauchy stress tensor is obtained from equation $(5)_{2}$ and has the explicit form

$$
\begin{aligned}
\boldsymbol{\sigma} & =2 W_{1} \mathbf{B}+2 W_{2}\left(I_{1} \mathbf{B}-\mathbf{B}^{2}\right)+2 W_{4} \mathbf{m} \otimes \mathbf{m}+2 W_{5}(\mathbf{m} \otimes \mathbf{B m}+\mathbf{B m} \otimes \mathbf{m}) \\
& +2 W_{6} \mathbf{m}^{\prime} \otimes \mathbf{m}^{\prime}+2 W_{7}\left(\mathbf{m}^{\prime} \otimes \mathbf{B m}^{\prime}+\mathbf{B m}^{\prime} \otimes \mathbf{m}^{\prime}\right) \\
& +W_{8}\left(\mathbf{m} \otimes \mathbf{m}^{\prime}+\mathbf{m}^{\prime} \otimes \mathbf{m}\right)-p \mathbf{I},
\end{aligned}
$$

where $\mathbf{m}=\mathbf{F M}, \mathbf{m}^{\prime}=\mathbf{F M}^{\prime}$ and the notation $W_{i}=\partial W / \partial I_{i}$ applies for $i=1,2,4, \ldots, 8$ with no dependency on $I_{3}$.

The strain energy of materials with preferred directions is, in general, written as the sum of two contributions, one associated with the isotropic properties of the base matrix and the second with the anisotropy being generated by the embedded fibers. For an incompressible material we have

$$
W=W_{\text {iso }}\left(I_{1}, I_{2}\right)+W_{\text {fib }}\left(I_{4}, I_{5}, I_{6}, I_{7}, I_{8}\right),
$$

where the term $W_{\text {iso }}$ represents the isotropic matrix and $W_{\text {fib }}$ accounts for the directional reinforcements (Qiu and Pence, 1997; Holzapfel et al., 2000; Merodio and Ogden, 2005; Horgan and Saccomandi, 2005; Dorfmann et al., 2007, 2008). Based on the structure of fascia lata outlined in Section 3.1 and following the simplification suggested by Holzapfel et al. (2000), we reduce the number of invariants and consider the form

$$
W=W_{\text {iso }}\left(I_{1}\right)+W_{\text {fib }}\left(I_{4}, I_{6}\right),
$$

which still provides sufficient flexibility to capture the mechanical response of fascia lata. The use of the reduced formulation (11) allows to write the Cauchy stress (9) as

$$
\boldsymbol{\sigma}=2 W_{1} \mathbf{B}+2 W_{4} \mathbf{m} \otimes \mathbf{m}+2 W_{6} \mathbf{m}^{\prime} \otimes \mathbf{m}^{\prime}-p \mathbf{I} .
$$

\section{A specific material model}

The disorganized collagen matrix that connects the inner and outer layers of fascia lata and envelops all fascicles is considered isotropic. We therefore 
propose the exponential strain energy function due to Demiray (1972) to model the mechanical properties. It has the form

$$
W_{\text {iso }}=\frac{\mu_{\text {iso }}}{2 \alpha}\left\{\exp \left[\alpha\left(I_{1}-3\right)\right]-1\right\},
$$

where $\mu_{\text {iso }}$ denotes the shear stiffness of the matrix and $\alpha$ is a dimensionless material parameter. The anisotropic character of the tissue is described by

$$
W_{\text {fib }}=\frac{\mu_{\mathrm{t}}}{2 k_{\mathrm{t}}}\left\{\exp \left[k_{\mathrm{t}}\left(I_{4}-1\right)^{2}\right]-1\right\}+\frac{\mu_{\mathrm{l}}}{2 k_{\mathrm{l}}}\left\{\exp \left[k_{\mathrm{l}}\left(I_{6}-1\right)^{2}\right]-1\right\},
$$

where the subscripts $t$ and 1 refer, respectively, to material properties in the transverse and longitudinal directions of fascia lata. The parameters $\mu_{\mathrm{t}}$ and $\mu_{1}$ describe the degree of anisotropy and $k_{\mathrm{t}}$ and $k_{1}$ are two dimensionless parameters associated with the two directions.

To obtain explicit expressions of (12) we place the two preferred directions $\mathbf{M}$ and $\mathbf{M}^{\prime}$ parallel to the $\left(X_{1}, X_{2}\right)$ plane as

$$
\mathbf{M}=\cos \varphi \mathbf{e}_{1}-\sin \varphi \mathbf{e}_{2}, \quad \mathbf{M}^{\prime}=\cos \varphi^{\prime} \mathbf{e}_{1}+\sin \varphi^{\prime} \mathbf{e}_{2}
$$

where $\mathbf{e}_{1}$ and $\mathbf{e}_{2}$ denote the in-plane Cartesian unit vectors and $\varphi$ and $\varphi^{\prime}$ the angles formed by the two fiber directions with $\mathbf{e}_{1}$. We use the membrane approximation $\sigma_{33}=0$ to eliminate the hydrostatic pressure term $p$ and the incompressibility condition $\operatorname{det} \mathbf{F} \equiv 1$ to eliminate the term $F_{33}$ from the above equations. We further assume pure homogeneous deformation with $F_{11}=\lambda_{1}, F_{22}=\lambda_{2}$ and $F_{12}=F_{21}=0$, where $\lambda_{1}$ and $\lambda_{2}$ denote the principal stretches. This last assumption will be discussed in more detail in the concluding remarks.

The right Cauchy-Green deformation tensor $\mathbf{C}$, which for planar extension has the components given by equation (4), simplifies to

$$
\mathbf{C}=\left[\begin{array}{ccc}
\lambda_{1}^{2} & 0 & 0 \\
0 & \lambda_{2}^{2} & 0 \\
0 & 0 & \lambda_{1}^{-2} \lambda_{2}^{-2}
\end{array}\right]
$$

with the invariant $I_{1}$ defined by $(6)_{1}$ given by

$$
I_{1}=\lambda_{1}^{2}+\lambda_{2}^{2}+\lambda_{1}^{-2} \lambda_{2}^{-2} .
$$

The use of equations (15) and (16) in (7) $)_{1,3}$ gives the invariants $I_{4}$ and $I_{6}$ as

$$
I_{4}=\lambda_{1}^{2} \cos ^{2} \varphi+\lambda_{2}^{2} \sin ^{2} \varphi, \quad I_{6}=\lambda_{1}^{2} \cos ^{2} \varphi^{\prime}+\lambda_{2}^{2} \sin ^{2} \varphi^{\prime} .
$$


Finally, using the strain energy functions (13) and (14) in (12) results in explicit expressions of the in-plane stress components. These are

$$
\begin{aligned}
\sigma_{11} & =\mu_{\text {iso }}\left(\lambda_{1}^{2}-\lambda_{1}^{-2} \lambda_{2}^{-2}\right) \exp \left[\alpha\left(I_{1}-3\right)\right] \\
& +2 \mu_{\mathrm{t}} \lambda_{1}^{2} \cos ^{2} \varphi\left(I_{4}-1\right) \exp \left[k_{\mathrm{t}}\left(I_{4}-1\right)^{2}-1\right] \\
& +2 \mu_{1} \lambda_{1}^{2} \cos ^{2} \varphi^{\prime}\left(I_{6}-1\right) \exp \left[k_{1}\left(I_{6}-1\right)^{2}-1\right], \\
& \\
\sigma_{22} & =\mu_{\text {iso }}\left(\lambda_{2}^{2}-\lambda_{1}^{-2} \lambda_{2}^{-2}\right) \exp \left[\alpha\left(I_{1}-3\right)\right] \\
& +2 \mu_{\mathrm{t}} \lambda_{2}^{2} \sin ^{2} \varphi\left(I_{4}-1\right) \exp \left[k_{\mathrm{t}}\left(I_{4}-1\right)^{2}-1\right] \\
& +2 \mu_{1} \lambda_{2}^{2} \sin ^{2} \varphi^{\prime}\left(I_{6}-1\right) \exp \left[k_{1}\left(I_{6}-1\right)^{2}-1\right],
\end{aligned}
$$

and

$$
\begin{aligned}
\sigma_{12} & =2 \lambda_{1} \lambda_{2}\left\{\mu_{\mathrm{t}} \cos \varphi \sin \varphi\left(I_{4}-1\right) \exp \left[k_{\mathrm{t}}\left(I_{4}-1\right)^{2}-1\right]\right. \\
& \left.+\mu_{1} \cos \varphi^{\prime} \sin \varphi^{\prime}\left(I_{6}-1\right) \exp \left[k_{1}\left(I_{6}-1\right)^{2}-1\right]\right\} .
\end{aligned}
$$

Equation (21) shows that, in general, shear stresses are required to maintain a pure homogeneous deformation. These vanish when the preferred directions coincide with the coordinate axes, i.e. $\varphi=0, \varphi^{\prime}=\pi / 2$. See the discussion of fiber-reinforced materials given by Ogden (2001).

\subsection{Model results}

The averaged response of the biaxial data of the fifth re-loading with maximum stretch $\lambda_{2}=1.06$ are represented by the dots in Figures 10 and 11 . More specifically, images (a) and (b) in Figure 10 show the average of the fifth loading cycle for specimens stretched in the direction of the longitudinally oriented fibers to the maximum stretch $\lambda_{2}=1.06$ for lateral stretches $\lambda_{1}=1$ and $\lambda_{1}=1.03$, respectively. The corresponding average data for specimens stretched in the direction of transversely oriented fibers are shown by the images (a) and (b) in Figure 11.

In what follows, we use the unit vector $\mathbf{M}^{\prime}$ to specify the fiber orientation that coincides with the loading-unloading direction. From equation (15) and using the sign convention introduced in Section 2.3 we find the fiber angle $\varphi^{\prime}=\pi / 2$, see Figure 2. The angle $\varphi$ that defines the orientation of the second family of fibers is considered a phenomenological parameter and is determined using a nonlinear optimization routine.

The nonlinear iterative algorithm for parameter estimation proposed by Buzzi-Ferraris and Manenti (2009) is applied to determine the non-negative 
Table 1: Values of the parameters used to define the isotropic energy functions (13), the anisotropic contribution (14) and the orientation of the preferred directions (15). The values of $\mu_{\mathrm{iso}}, \mu_{\mathrm{l}}, \mu_{\mathrm{t}}$ are given in $\mathrm{MPa}$, the angle $\varphi$ in degrees.

\begin{tabular}{|c|c|c|c|c|c|c|}
\hline \multicolumn{7}{|c|}{ Summary of model parameters. } \\
\hline$\mu_{\text {iso }}$ & $\alpha$ & $\mu_{\mathrm{l}}$ & $k_{\mathrm{l}}$ & $\mu_{\mathrm{t}}$ & $k_{\mathrm{t}}$ & $\varphi$ \\
\hline 4.8597 & 25.00 & 32.5193 & 60.6070 & 2.9505 & 60.7569 & 22.1982 \\
\hline
\end{tabular}

values of the parameters $\mu_{\text {iso }}, \alpha, \mu_{\mathrm{l}}, k_{\mathrm{l}}, \mu_{\mathrm{t}}, k_{\mathrm{t}}, \varphi$ (see Table 1 ). The equations (19) and (20) are used in conjunction with the data in the minimization routine to determine the set of parameters returning the smallest residuals between the predicted and measured responses.

To limit the searchable domain of parameter sets giving accurate and stable solutions we use the results from the tissue structure obtained from histology and guide the range of permitted values for $\mu_{\mathrm{l}}, \mu_{\mathrm{t}}$ and $\varphi$. Precisely, based on the fiber bundle longitudinal to transverse cross sectional area evaluation, the value of the stiffness for $\mu_{\mathrm{t}}$ is limited to be no less than one order of magnitude smaller than the permitted value for $\mu_{1}$, likewise the permitted range of solutions for the fiber angle $\varphi$ between the two layers is given a lower bound of $10^{\circ}$ and an upper bound of $25^{\circ}$. We recall that $\varphi$ denotes the fiber angle with respect to the 1 -axis and therefore the value of $\left(90^{\circ}-\varphi\right)$ compares with the opening angle reported in Section 3.

The values of the material parameters summarized in Table 1 are used to evaluate the in-plane Cauchy stress components given by equations (19), (20) and (21). As a consequence of the preferred directions not being orthogonal, i.e. $\varphi \neq 0$, the shear stress component $\sigma_{12}$ is different from zero. Using equation (21), $\sigma_{12}$ is approximately an order of magnitude smaller than $\sigma_{11}$ and $\sigma_{22}$. Since no data of the in-plane shear stress exist, we do not include the graphs of $\sigma_{12}$.

With the material parameters given in Table 1 the model is now used to determine the stress components $\sigma_{11}$ and $\sigma_{22}$ in biaxial tension. Figure 10 compares the numerical results, shown by solid lines, to the averaged data of fascia lata stretched in the direction of the longitudinally oriented fibers. Image (a) illustrates the material and model responses when $\lambda_{1}=$ 1 and constant and the stretch in the 2-direction increases from $\lambda_{2}=1$ 

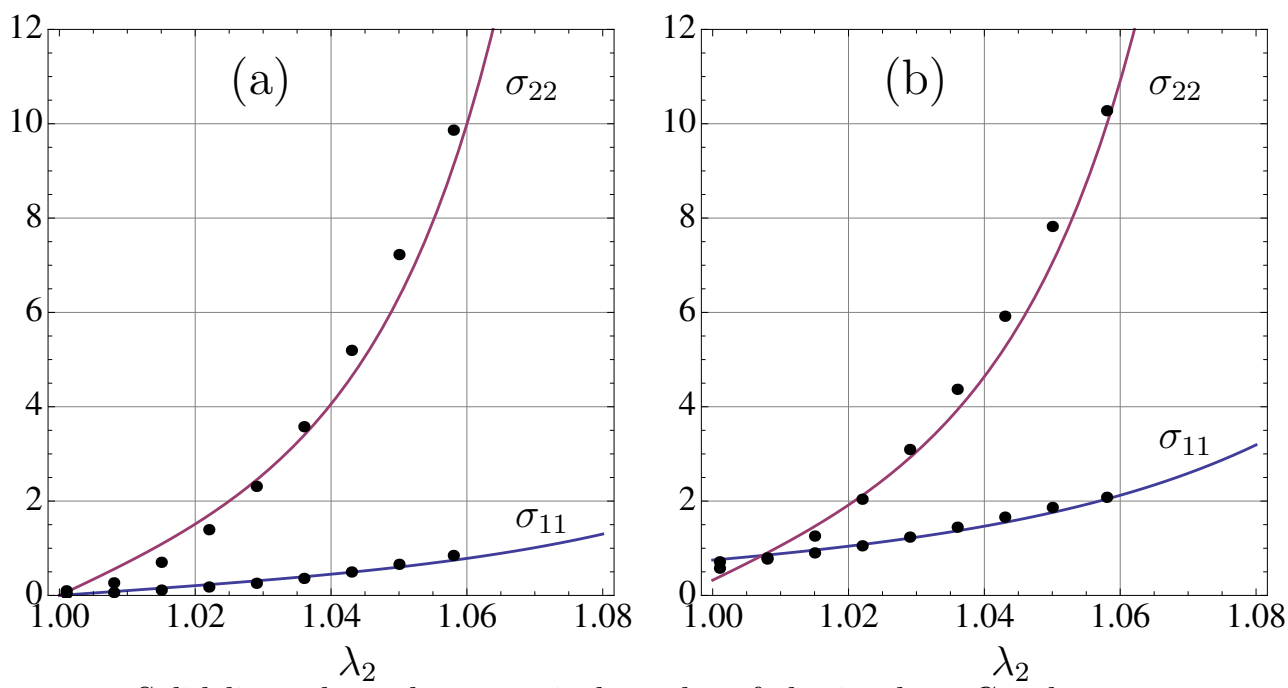

Figure 10: Solid lines show the numerical results of the in-plane Cauchy stress components $\sigma_{11}$ and $\sigma_{22}$, dotted lines the corresponding averaged experimental data. Image (a) illustrates the response when $\lambda_{1}=1$ and the stretch in the longitudinally oriented fiber direction increases to $\lambda_{2}=1.08$. Graphs in (b) show the corresponding results when the stretch in the 1-direction is increased to a constant value of $\lambda_{1}=1.03$. The values of the Cauchy stress components are given in MPa.

to a maximum value of $\lambda_{2}=1.08$. The graphs in image (b) again show the material and numerical responses during the initial extension in the 1direction to $\lambda_{1}=1.03$ with $\lambda_{2}=1$ constant. Then, with $\lambda_{1}=1.03$ and constant, the material is again stretched in the longitudinal fiber direction to $\lambda_{2}=1.08$. The results show the model accurately describes the experimental behavior of the tissue.

Similarly, Figure 11 shows the averaged data in biaxial tension of fascia lata when stretched in the direction of the transversely oriented fibers. The numerical solution again shows good agreement with the experimental data.

The model developed in Section 5 is now specialized to uniaxial tension. In this context it is important to underscore that the values of the material parameters in Table 1 are obtained using only the biaxial tension data. The numerical results are therefore of particular interest since they provide the opportunity to test the accuracy of the numerical response. The results of the constitutive model for tensile loading in the direction of longitudinally and transversely oriented fibers are shown by solid lines in Figure 5. Good agreement with the experimental data is obtained up to values of $1.06 \%$ in the two directions. Upon reaching this stretch, the stiffening behavior changes 

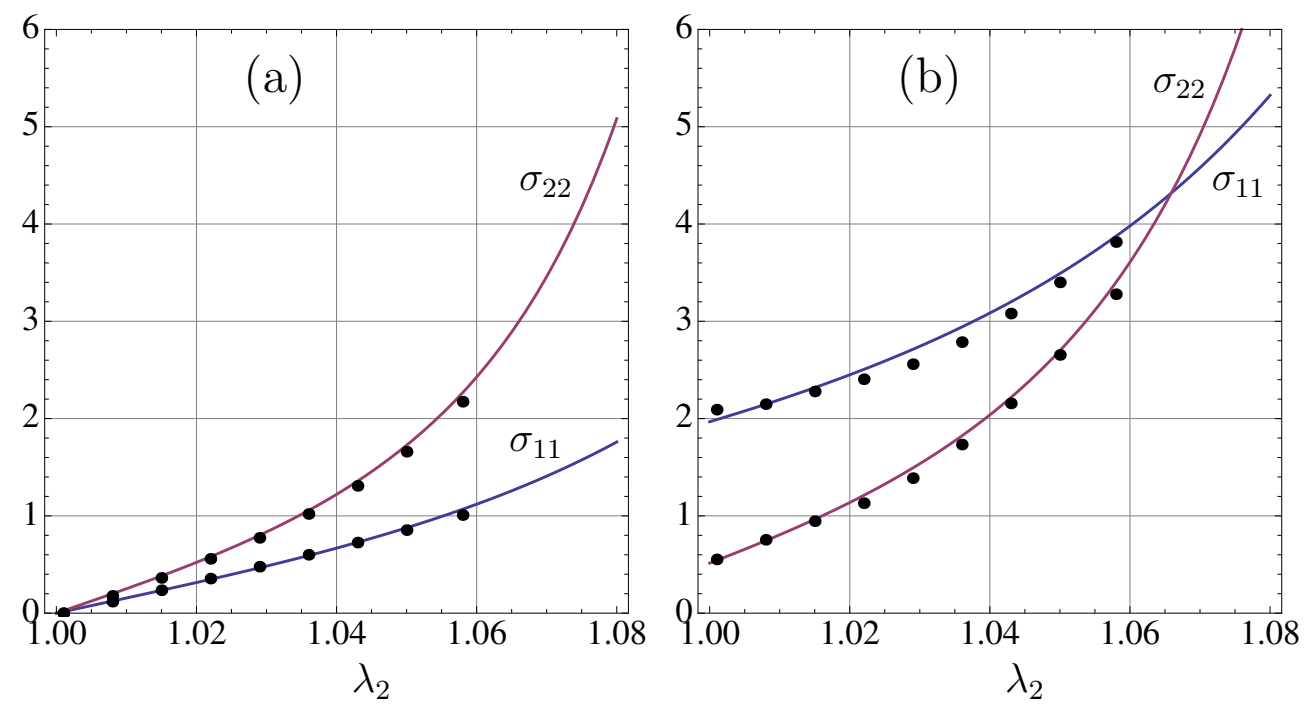

Figure 11: Solid lines show the numerical results of the in-plane Cauchy stress components $\sigma_{11}$ and $\sigma_{22}$, dotted lines the corresponding averaged experimental data. Image (a) illustrates the response when $\lambda_{1}=1$ and the stretch in the transversely oriented fiber direction increases to $\lambda_{2}=1.08$. Graphs in (b) show the corresponding results when the stretch in the 1-direction is increased to a constant value of $\lambda_{1}=1.03$. The values of the Cauchy stress components are given in MPa.

indicating the onset of softening due to alterations in the microstructure of the tissue. Therefore, the elastic limit in uniaxial tension of fascia lata is $\approx 6 \%$.

\section{Concluding remarks}

Motivated by the need to characterize the nonlinear mechanical properties of connective tissue, such as fascia lata, we have presented new experimental data and developed a mathematical model that captures the observed behavior with good accuracy. The experimental data, in addition to simple tension, include planar biaxial extension that more closely resemble fascia's in vivo state of strain.

The histological analysis of goat fascia lata showed a bi-layered structure containing collagen fascicles oriented along two preferred directions providing anisotropic material behavior. The arrangement and the cross-sectional areas of the longitudinally and transversely oriented fascicles are different and therefore the two directions are not mechanically equivalent nor are they 
orthogonal. Then, the in-plane shear stress (21) is required for the pure homogeneous deformation (16), that is, the principal axes of stress and strain do not coincide. However, to our knowledge, there is currently no experimental equipment available that allows applications of in-plane shear stress to a soft biological tissue. In the absence of in-plane shear stress $\sigma_{12}$, the off-diagonal terms $F_{12}$ and $F_{21}$ in equation (2) are different from zero. Therefore, in our approximation we do not include the effects of in-plane shear deformation.

The effect of in-plane shear is quantified in the following. Image (a) in Figure 12 defines the undeformed configuration $\lambda_{1}=\lambda_{2}=1$ from the video extensometer using the physical gage marks to outline a rectangular reference area. Image (b) depicts the sample stretched in the direction of the transversely oriented fibers to $\lambda_{2}=1.06$ with $\lambda_{1}=1.03$ constant. The solid lines indicate the size of the reference area, the dashed lines define the deformed shape tangent to the physical gage marks. The intersections of the dotted lines with the physical gage marks define the points that are used by the equipment to control strain. Image (b) compares the rectangular reference area to the current shape and is used to measure an in-plane shear of 0.0872 rad. For completeness, we recall that the corresponding stress-deformation data are shown by Figure 9(d). Therefore, specializing the constitutive theory in Section 5 to pure homogeneous deformation is, in this special case, an acceptable approximation. For a more general discussion of the effects of in-plane shear during biaxial testing of biological tissues we refer to, for example, Sacks (2000).

\section{References}

Bennett, M. B., 1989. A possible energy-saving role for the major fascia of the thigh in running quadrupedal mammals. Trans. Zool. Soc. London 219 (Part 2), 221-230.

Butler, D. L., Grood, E. S., Noyes, F. R., Zernicke, R. F., Brackett, K., 1984. Effects of structure and strain-measurement technique on the material properties of young human tendons and fascia. J. Biomech. 17 (8), $579-596$.

Buzzi-Ferraris, G., Manenti, F., 2009. Kinetic models analysis. Chem. Eng. Sci. 64 (5), 1061-1074. 


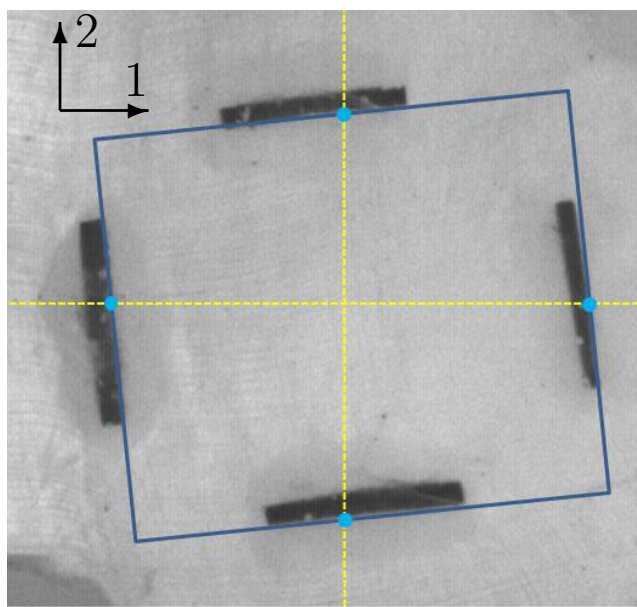

(a)

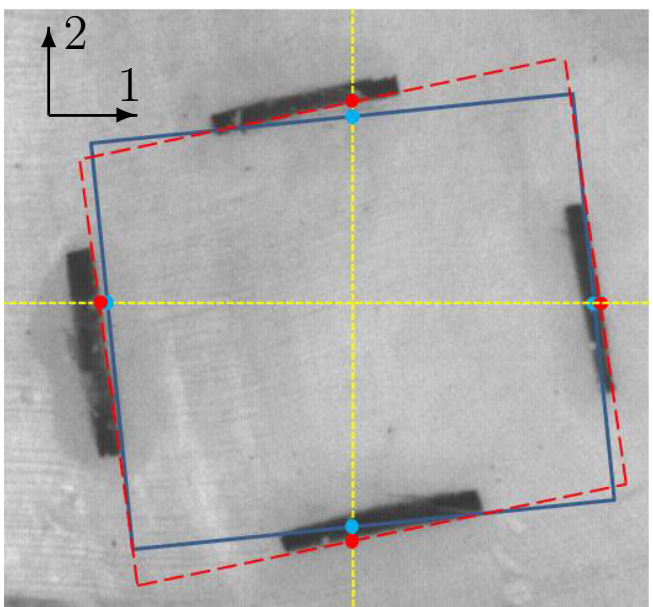

(b)

Figure 12: The rectangular reference area $\lambda_{1}=\lambda_{2}=1$ is defined by (a). Image (b) shows the deformed sample stretched in the direction of the transversely oriented fibers to $\lambda_{2}=1.06$ with $\lambda_{1}=1.03$ constant. The intersections of the dotted lines with the physical gage marks define the points used by the equipment to control strain. In (b) the rectangular reference area is compared to the deformed shape, which is defined by dashed lines. The amount of in-plane shear in (b) is equal to $0.0872 \mathrm{rad}$.

Demiray, H., 1972. A note on the elasticty of soft biological tissues. J. Biomech. 5, 309-311.

Dorfmann, A., Trimmer, B. A., Woods, W. A., 2007. A constitutive model for muscle properties in a soft-bodied arthropod. J. Roy. Soc. Interface 4, $257-269$.

Dorfmann, A. L., Woods, W. A., Trimmer, B. A., 2008. Muscle performance in a soft-bodied terrestrial crawler: constitutive modeling of strain-rate dependency. J. Roy. Soc. Interface 5, 349-362.

Eng, C. M., Pancheri, F., Lieberman, D. E., Biewener, A. A., Dorfmann, A. L., 2013. Biaxial material properties of goat fascia lata. J. Biomech., submitted.

Fung, Y. C., 1967. Elasticity of soft tissues in simple elongation. Am. J. Physiol. 213, 1532-1544.

Garfin, S. R., Tipton, C. M., Mubarak, S. J., Woo, S., Hargens, A. R., Akenson, W. H., 1981. Role of fascia in maintenance of muscle tension and pressure. J. Appl. Physiol. 51 (2), 317-320. 
Gratz, C. M., 1931. Tensile strength and elasticity tests on human fascia lata. J. Bone Joint Surg. 13, 334-340.

Haut, R. C., Little, R. W., 1972. A constitutive equation for collagen fibers. J. Biomech. 5 (5), 423-\&.

Hinton, R., Jinnah, R. H., Johnson, C., Warden, K., Clarke, H. J., 1992. A biomechanical analysis of solvent-dehydrated and freeze-dried human fascia lata allografts - a preliminary report. Am. J. Sport. Med. 20 (5), 607-612.

Holzapfel, G. A., 2001. Nonlinear Solid Mechanics: A Continuum Approach for Engineering. John Wiley \& Sons, Chichester.

Holzapfel, G. A., Gasser, T. C., Ogden, R. W., 2000. A new constitutive framework for arterial wall mechanics and a comparative study of material models. J. Elasticity 61, 1-48.

Holzapfel, G. A., Ogden, R. W., 2009. On planar biaxial tests for anisotropic nonlinearly elastic solids. A continuum mechanical framework. Math. Mech. Solids 14 (5), 474-489.

Horgan, C. O., Saccomandi, G., 2005. A new constitutive theory for fiberreinforced incompressible nonlinearly elastic solids. J. Mech. Phys. Solid 53, 1985-2015.

Jenkins, R. B., Little, R. W., 1974. A constitutive equation for parallellfibered elastic tissue. J. Biomech. 7, 397-402.

Lanir, Y., 1983. Constitutive-equations for fibrous connective tissues. J. Biomech. 16 (1), 1-12.

Lee, D., Glueck, M., Khan, A., Fiume, E., Jackson, K., 2010. A survey of modeling and simulation of skeletal muscle. Acm T. Graphic. 28 (4).

Lin, H.-T., Dorfmann, A. L., Trimmer, B. A., 2009. Soft-cuticle biomechanics: constitutive model of anisotropy for caterpillar integument. J. Theor. Biol. 256, 447-457. 
Maas, H., Huijing, P. A., 2005. Myofascial force transmission in dynamic muscle conditions: effects of dynamic shortening of a single head of multitendoned rat extensor digitorum longus muscle. Eur. J. Appl. Physiol. 94 (5-6), 584-592.

Maas, H., Lehti, T. M., Tiihonen, V., Komulainen, J., Huijing, P. A., 2005a. Controlled intermittent shortening contractions of a muscle-tendon complex: muscle fibre damage and effects on force transmission from a single head of rat edl. J. Muscle Res. Cell. M. 26 (4-5), 259-273.

Maas, H., Meijer, H. J. M., Huijing, P. A., 2005b. Intermuscular interaction between synergists in rat originates from both intermuscular and extramuscular myofascial force transmission. Cells Tissues Organs 181 (1), 38-50.

Merodio, J., Ogden, R. W., 2005. Mechanical response of fiber-reinforced incompressible non-linear elastic solids. Int. J. Nonlinear Mech. 40, 213227.

Natali, A. N., Pavan, P. G., Stecco, C., 2010. A constitutive model for the mechanical characterization of the plantar fascia. Connect. Tissue. Res. 51 (5), 337-346.

Ogden, R. W., 1997. Non-linear Elastic Deformations. Dover Publications, New York.

Ogden, R. W., 2001. Elements of the theory of finite elasticity. In: Fu, Y. B., Ogden, R. W. (Eds.), Nonlinear Elasticity: Theory and Applications. Cambridge University Press, pp. 1-58.

Pancheri, Q. P., Dorfmann, A., 2013. Strain controlled biaxial tension of natural rubber: New experimental data. Rubber Chem. Technol. in press.

Qiu, G. Y., Pence, T. J., 1997. Remarks on the behavior of simple directionally reinforced incompressible nonlinearly elastic solids. J. Elasticity 49, $1-30$.

Sacks, M. S., 2000. Biaxial mechanical evaluation of planar biological materials. J. Elasticity 61 (1-3), 199-246.

Sacks, M. S., Sun, W., 2003. Multiaxial mechanical behavior of biological materials. Ann. Biomed. Eng. 5, 251-284. 
Spencer, A. J. M., 1971. Theory of invariants. In: Eringen, A. C. (Ed.), Continuum Physics. Vol. 1. Academic Press, New York, pp. 239-353.

Stecco, C., Pavan, P. G., Porzionato, A., Macchi, V., Lancerotto, L., Carniel, E. L., Natali, A. N., De Caro, R., 2009. Mechanics of crural fascia: from anatomy to constitutive modelling. Surg. Radiol. Anat. 31 (7), 523-529.

Tang, C. Y., Zhang, G., Tsui, C. P., 2009. A 3d skeletal muscle model coupled with active contraction of muscle fibres and hyperelastic behaviour. J. Biomech. 42 (7), 865-872.

Veronda, D. R., Westmann, R. A., 1970. Mechanical characterization of skin - finite deformations. J. Biomech 3 (1), 111-124. 